\title{
Structural basis for the multimerization of nonstructural protein nsp9 from SARS-CoV-2
}

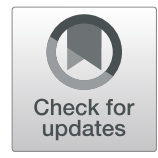

\author{
Changhui Zhang ${ }^{1}$, Yiping Chen', Li Li ${ }^{1}$, Yan Yang ${ }^{1}$, Jun $\mathrm{He}^{2}$, Cheng Chen ${ }^{3}$ and Dan Su ${ }^{* *}$
}

\begin{abstract}
Severe acute respiratory syndrome coronavirus-2 (SARS-CoV-2), the causative agent of a potentially fatal disease named coronavirus disease 2019 (COVID-19), has raised significant public health concerns globally. To date, the COVID-19 pandemic has caused millions of people to be infected with SARS-CoV-2 worldwide. It has been known since the 2003 SARS epidemic that coronaviruses (CoVs) have large RNA genomes, the replication of which requires an RNA-dependent RNA replication/transcription complex. CoV nonstructural proteins (Nsps) play pivotal roles in the assembly of this complex and associated enzymatic functions in virus genomic replication. Several smaller nonenzymatic Nsps assist with RNA-dependent RNA polymerase function. In this study, we determined the structure of SARS-CoV-2 nonstructural protein 9 (nsp9), an RNA-binding protein that is essential for CoV replication. Its homotetrameric structure with two stable dimeric interfaces provids a structural basis for understanding the mechanisms of RNA-binding protein self-assembly, which may be essential for the regulation of viral RNA replication and transcription.
\end{abstract}

Keywords: SARS-CoV-2, COVID-19, nsp9, Tetramer

\section{Introduction}

Coronavirus disease 2019 (COVID-19), an acute respiratory distress syndrome caused by severe acute respiratory syndrome coronavirus-2 (SARS-CoV-2), is currently a global pandemic that has been spreading across all continents since late 2019 [1-3]. According to the World Health Organization COVID-19 situation reports, the number of individuals with a confirmed SARS-CoV2 infection stood at approximately 16 million worldwide as of July 27, 2020, with related fatalities standing at over 0.64 million [4]. SARS-CoV-2 is a coronavirus (CoV) strain that belongs to the family Coronaviridae [5]. Genomic analysis suggests that SARS-CoV-2 is a new member of the genus Betacoronavirus, which is most closely related to the SARS-like virus previously identified in wild bats [6]. Human betacoronaviruses, including SARS-CoV, Middle East respiratory syndrome

\footnotetext{
* Correspondence: sudan@scu.edu.cn

'State Key Laboratory of Biotherapy, West China Hospital, Sichuan University, and Collaborative Innovation Center for Biotherapy, Chengdu 610041, PR

China

Full list of author information is available at the end of the article
}

coronavirus (MERS-CoV), and SARS-CoV-2, share more sequence similarities with one another than with other CoVs [7]. However, SARS-CoV-2 has very high transmissibility, exhibiting rapid spreading through humanto-human contact, causing the WHO to declare it as a worldwide health emergency [8].

The genome of SARS-CoVs consists of an approximately 30-kb non-segmented positive-sense RNA sequence with a $5^{\prime}$ untranslated region (UTR), followed by a single open reading frame and a short flanking 3' UTR [9]. The life cycle of SARS-CoVs starts with its binding to the cell surface receptor angiotensin-converting enzyme 2 (ACE2) through its spike glycoproteins, resulting in receptor-mediated endocytosis [10]. Upon entry into the host cell, the virus expresses its replicase gene to initially generate two precursor polyproteins, ppla and pplab. These then undergo cleavage to yield 16 mature nonstructural proteins (nsp1-16) as well as several intermediate precursors by two distinct viral proteinases, the papain-like proteinase within nsp3 and a 3C-like proteinase nsp5 [11]. These nsps includes replicative enzymes (e.g., nsp12/RdRp, and nsp13/helicase), a variety 
of subunits performing accessory functions for viral RNA synthesis (e.g., nsp8/primase, nsp14/exoribonuclesae, and nsp15/endoribonuclease NendoU) and several smaller nonenzymatic nsps (nsp7, nsp9, and nsp10) assist with RNA-dependent RNA polymerase function [12-17].

As a member of the oligosaccharide/oligonucleotidebinding (OB-fold) superfamily, the RNA-binding protein nsp9 is critical to the RNA-dependent RNA replicase machinery of CoVs [18] nsp9 is essential for efficient viral growth as it plays a pivotal role in the formation of the replication and transcription complex machinery [19]. Mutation of the SARS-CoV $n s p 9$ gene through a reverse genetics approach was found to prevent the propagation of the virus [20]. The sequences of nsp9 homologs are highly conserved among betacoronaviruses especially between SARS-CoV and SARS-CoV-2 ( 97\% sequence homology), and several nsp9 homologous structures have been determined in different viral species, including SARS-CoV [19, 21-24].

To elucidate the molecular mechanisms of SARS$\mathrm{CoV}-2$ replication, which would aid in the diagnosis, treatment, and prevention of the COVID-19 pandemic, researchers have made significant efforts to characterize the Nsp structures in a short amount of time since the COVID-2019 outbreak [18]. In this paper, we report the crystal structure of SARS-CoV-2 nsp9 at $2.95 \AA$. This structure was revealed to be a horseshoe-like tetramer, which may play an essential role in nsp9 oligomerization and in the regulation of viral nucleic acid binding during the replication of the virus. Two significant contact surfaces stabilize the SARS-CoV-2 nsp9 tetramer. One interface is composed of a parallel association of the Cterminal alpha $(\alpha)$-helices and the $\mathrm{N}$-terminal loop, which are highly conserved across $\mathrm{CoV}$ species. Antiparallel beta $(\beta)$-strands form the other interface from both protomers of nsp9, zippering the two $\beta$-barrels together. The structure of SARS-CoV-2 nsp9 provides insights into its multimerization and the regulation of viral nucleic acid binding during $\mathrm{CoV}$ replication.

\section{Results}

\section{Overall structure of the SARS-CoV-2 nsp9 protomer}

The SARS-CoV-2 nsp9 protomer is made up of seven $\beta$ strands $\left(\beta_{1}-\beta_{7}\right)$ flanked by an $\mathrm{N}$-terminal extension and a C-terminal $\alpha$-helix $\left(\alpha_{1}\right)$ (Fig. 1a). The core of the protein comprises a $\beta$-stranded barrel made up of two antiparallel $\beta$-sheets that are packed orthogonally. One of the sheets is formed by $\beta_{1}-\beta_{3}$ with a half strand of $\beta_{7}$, and the other sheet is composed of the $\beta_{4}$ and $\beta_{5}$ strands. A tight $\beta$-hairpin involving $\beta_{6}$ and $\beta_{7}$ lies adjacent to the $\alpha$-helix, extending out of the $\beta$-barrel. The elongated loops link the individual $\beta$-sheets of the barrel, along with the $\mathrm{N}$-terminal $\beta$-strand and a C-terminal $\alpha$ - helix. The fold of this nsp9 protomer meets the structural features of OB-fold modules, one of which is a $\beta$ barrel consisting of six highly coiled antiparallel $\beta$-sheets [25]. Therefore, the structural assignment of the nsp9 monomer is the OB-fold, a compact structural motif in the OB-fold superfamily that is frequently used for nucleic acid recognition [26].

The SARS-CoV-2 nsp9 protomeric structure is highly conserved among CoVs. To date, five nsp9 structures from various $\mathrm{CoV}$ species have been reported; namely, SARS-CoV nsp9 (PDB: 1QZ8), avian infectious bronchitis virus (IBV) nsp9 (PDB: 5C94), human coronavirus (hCoV) 229E nsp9 (PDB: 2 J97), porcine delta coronavirus (PDCoV) nsp9 (PDB: 5YM6), and porcine epidemic diarrhea virus (PEDV) nsp9 (PDB: 5HIZ) [21-24]. A superimposition of all protomeric nsp9 backbone coordinates showed that the root-mean-square deviation values between SARS-CoV-2 and SARS-CoV, IBV, $\mathrm{HCoV}, \mathrm{PDCoV}$, and PDEV are $0.562 \AA$ for $88 \mathrm{C}_{\alpha}$ atoms, $0.70 \AA$ for $73 \mathrm{C}_{\alpha}$ atoms, $1.311 \AA$ for $50 \mathrm{C}_{\alpha}$ atoms, 3.940 $\AA$ for $74 C_{\alpha}$ atoms, and $0.479 \AA$ for $52 C_{\alpha}$ atoms, respectively (Fig. $1 \mathrm{~b}-\mathrm{f}$ ). These data suggest that the nsp9 structure of SARS-Cov-2 is highly similar to that of all CoVs, especially SARS-CoV nsp9 (Fig. 1g).

\section{Structural basis for SARS-CoV-2 nsp9 multimerization}

The initial structure solved by molecular replacement showed that six SARS-CoV-2 nsp9 protomers form an OB-fold cluster in an asymmetric unit (Supplementary Fig. 1a). The OB-fold cluster is stacked in three layers (I, II, and III). For the convenience of description, we have named the protomer in layer I as molecule a', the three protomers in layer II as molecule $\mathrm{b}^{\prime} / \mathrm{c}^{\prime} / \mathrm{d}^{\prime}$, and the two protomers in layer III as molecule e'/f '. To obtain more information about the protein interfaces and the likely biological assemblies of the OB-fold cluster, we calculated the structure of SARS-CoV-2 nsp9 using PDBePISA [27]. The buried area and binding energy results (Supplementary Table 1) indicated that there is one stable tetrameric form of SARS-CoV-2 nsp9 in the crystal lattice. Each protomer associated with the tetramer assembly comes from different asymmetric units in this structure (Supplementary Fig. 1b). In order to get the best view of the tetramer of nsp 9 and to provid a general description of the structure, we refined the structure to get a new asymmetry unit that contains an intact tetrameric form of nsp9. In the new asymmetric unit, four of six protomers form a stable tetramer (molecule $\mathrm{a} / \mathrm{b} / \mathrm{c} / \mathrm{d}$ ) (Fig. 2a) while the other two protomers on the side of the tetramer, which forms tetramer with other two protomers in neighbor unit. Overall, four nsp9 protomers assemble into a horseshoe-like complex, with a buried area of close to $4638 \AA^{2}$ (Fig. $2 b$ and c). 


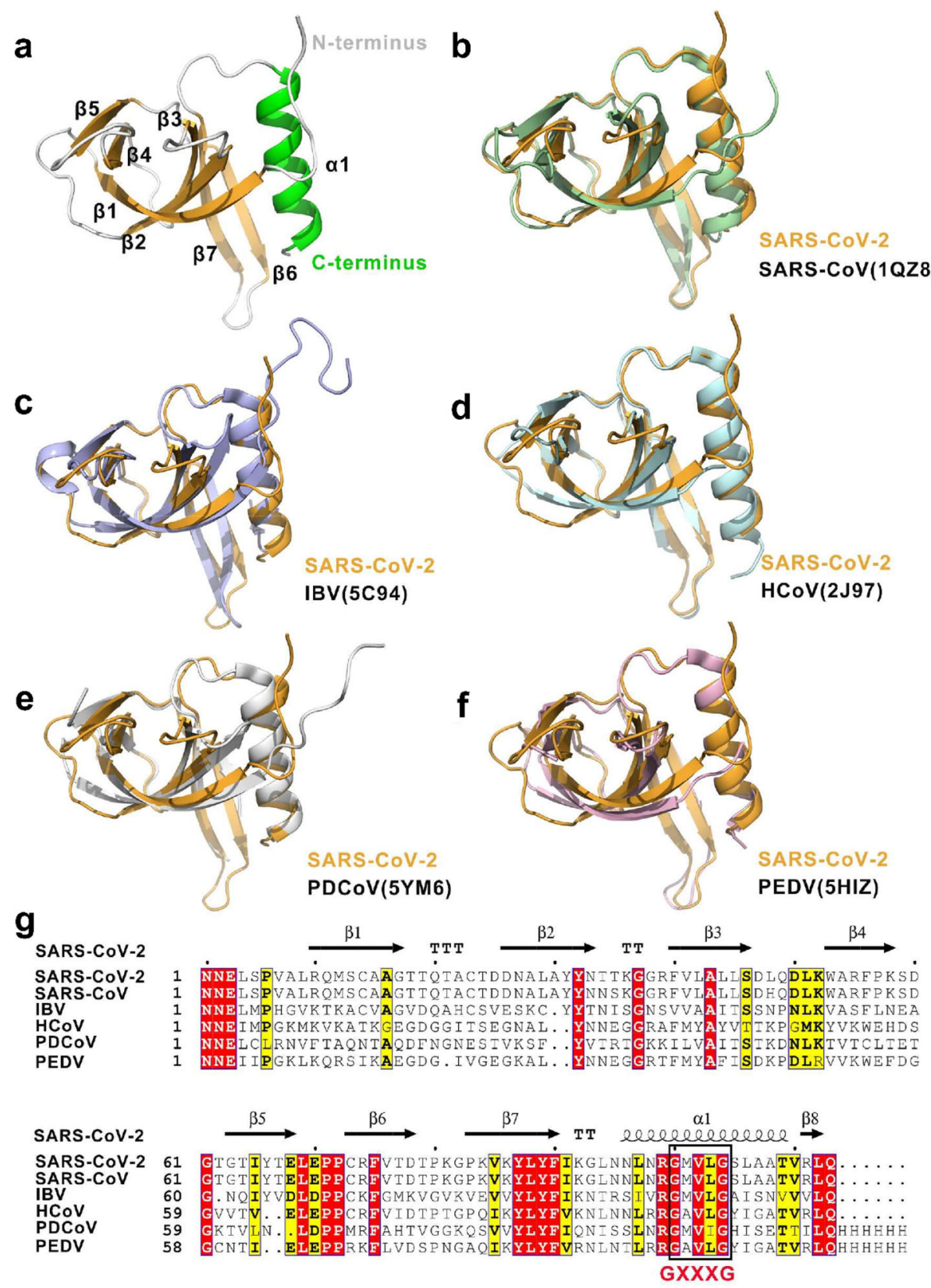

Fig. 1 Overall structure of the SARS-CoV-2 nsp9 protomer. a Different views of the protomeric architecture of SARS-CoV-2 nsp9 with the secondary structure labeled. The a-helix, $\beta$-sheets, and loops are colored in green, orange, and white, respectively. b-f Superimposition of SARSCoV-2 nsp9 with other nsp9 structures in the coronavirus family. The nsp9s from SARS-CoV-2, SARS-CoV, avian infectious bronchitis virus (IBV), human coronavirus 229E (hCoV-229E), porcine delta coronavirus (PDCoV), and porcine epidemic diarrhea virus (PDEV) are colored in orange, light blue, marine, white, and salmon, respectively. The PDB codes are indicated in the lower right corner. gSequence alignment of CoV nsp9 homologs. Comparison of the SARS-CoV, IBV, HCoV, PDCoV, and PDEV sequences with the SARS-CoV-2 sequence. Identical residues are highlighted in red, and conserved residues are shown in yellow. The table was produced with ESPript 3.0, using secondary structure elements for SARS-CoV-2 nsp9 assigned using DSSP. Residues boxed in red are completely conserved

Three interfaces $\left(\mathrm{I}_{\mathrm{a} / \mathrm{b}}, \mathrm{I}_{\mathrm{b} / \mathrm{c}}\right.$, and $\left.\mathrm{I}_{\mathrm{c} / \mathrm{d}}\right)$ are engaged in the tetrameric structure in two combination modes (helix interface and sheet interface). Interfaces $I_{a / b}$ (between molecules a and b) and $\mathrm{I}_{\mathrm{c} / \mathrm{d}}$ (between molecules $\mathrm{c}$ and $\mathrm{d}$ ) share the same combination mode (i.e., the helix interface), having a buried area of $626 \AA^{2}$ and $652 \AA^{2}$, respectively. Each helix interface involves the conserved Cterminal $\alpha$-helices (residues 96-109) that are associated in parallel and the $\mathrm{N}$-terminus of nsp9 (residues 1-9) (Fig. 3a and b). The parallel $\alpha$-helices allow two 

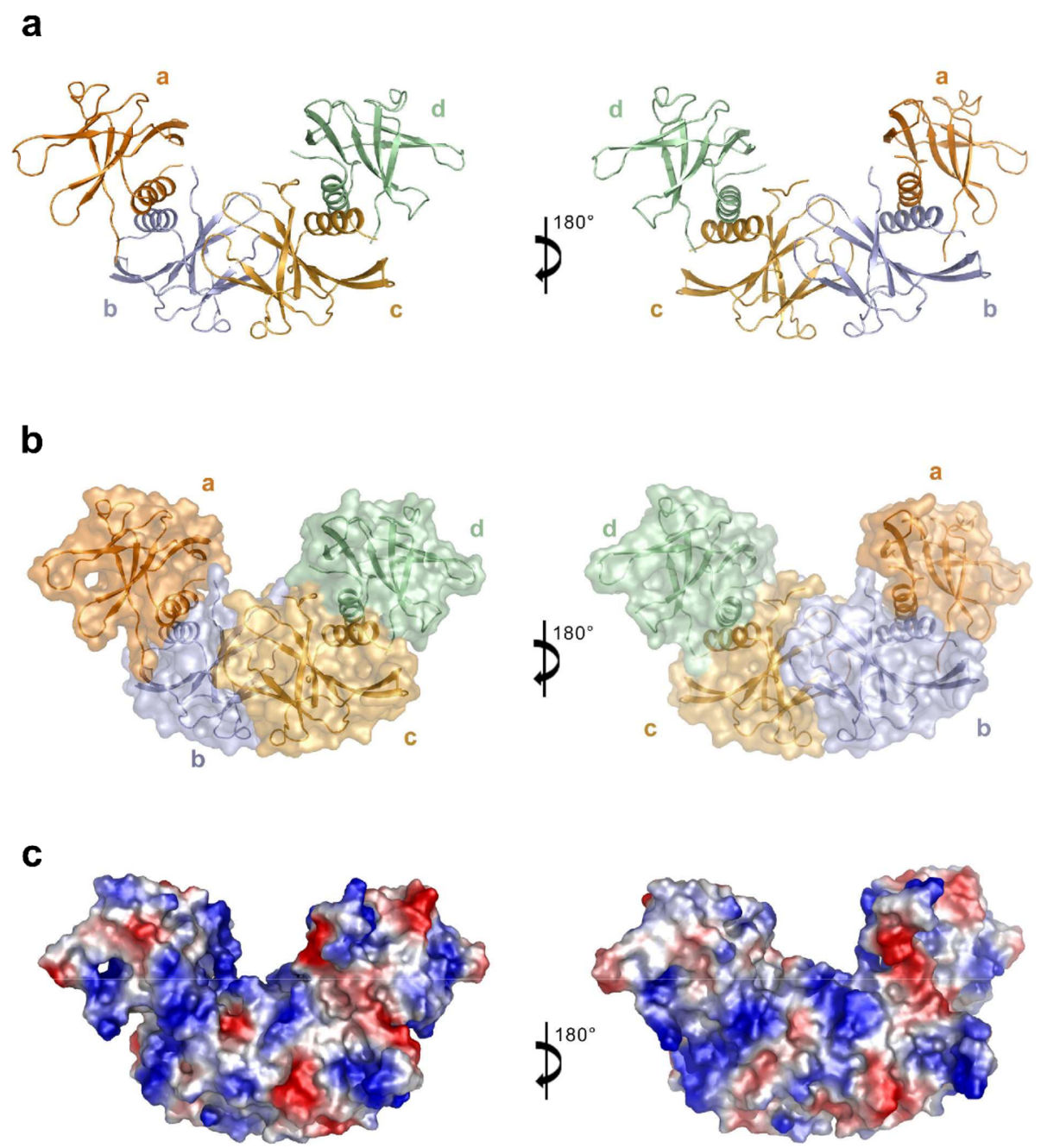

Fig. 2 Structure of the SARS-CoV-2 nsp9 tetramer. a Architecture and assembly of the nsp9 tetramer. Molecules a, b, c, and d in the stable tetramer are colored in orange, light blue, bright orange and pale green, respectively, and depicted in cartoon representations. $\mathbf{b}$ The stable tetramer is colored, labeled as Fig. $2 \mathrm{a}$ and depicted in cartoon and surface representations. c The electrostatic potential surface of the stable tetramer. Blue, positive charge $\left(+70 \mathrm{k}_{B} \mathrm{~T}\right)$; Red, negative charge $\left(-70 \mathrm{k}_{\mathrm{B}} \mathrm{T}\right)$

molecules to be packed together by a conserved protein-protein interaction motif (GXXXG). Residues G100 and G104, which are located on the helices from each subunit, play a pivotal role in tetramer stabilization. Further stabilization of this tetrameric interface is given by residues $6-10$ in the $\mathrm{N}$-terminus, clipping to the edge of $\beta_{6}$ from its neighboring partner.

Compared with previously reported $\mathrm{CoV}$ nsp9 structures, interface $\mathrm{I}_{\mathrm{b} / \mathrm{c}}$ (between molecules $\mathrm{b}$ and $\mathrm{c}$ ) is a unique binding surface located in the center of the SARS-CoV-2 nsp9 tetrameric structure (Fig. 3c). This interactive region adopts a sheet-interface style formed by $\beta_{5}$ (residues $64-68$ ) and three connection loops $\left(\mathrm{LP}_{1}\right.$, $\mathrm{LP}_{2}$, and $\mathrm{LP}_{3}$ ) from both molecules $\mathrm{b}$ and $\mathrm{c}$. The buried area of the $\mathrm{I}_{\mathrm{b} / \mathrm{c}}$ interface is $1040 \AA^{2}$, which is the largest buried area compared with those of interfaces $I_{c / d}$ and $\mathrm{I}_{\mathrm{a} / \mathrm{b}}$ in the SARS-CoV-2 nsp9 tetramer. There are three major contact regions in the $\mathrm{I}_{\mathrm{b} / \mathrm{c}}$ interface. The first contact region associates with two $\mathrm{LP}_{1}$ loops (residues 3342 ), connecting $\beta_{2}$ and $\beta_{3}$ from each of the subunits of $c$ and b. Part of the N-terminal tail (residues 2-5) from molecule $\mathrm{c}$ is also involved in this contact surface. Two hydrogen bonds and one salt bond are involved in the interface between residues E3 and T35 in molecule c and residue $\mathrm{K} 36$ in molecular b (Fig. 3d). Additionally, residues G38, F40, V41, and L44 make a significant contribution to this hydrophobic contact surface. The second contact region consists of connecting $\mathrm{LP}_{2}$ (residues 58-63) and $\mathrm{LP}_{3}$ loops (residues 91-96) and $\beta_{5}$ (residues 64-68) from each of the subunits. The $\mathrm{LP}_{2}$ loop on molecule c comes in direct contact with $\mathrm{LP}_{3}$ on molecule b. An antiparallel $\beta$-sheet located at the center of the sheet interface is composed of two $\beta_{5}$ strands. In this hydrophobic contact surface, three residues (T62, T64, and 
a

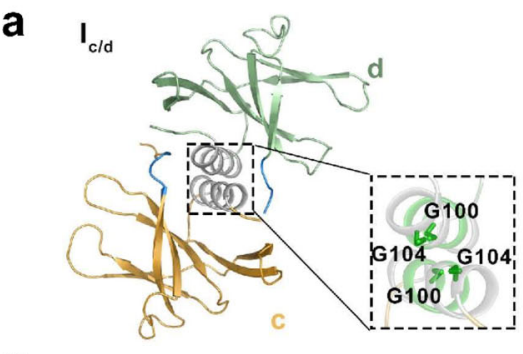

$\mathbf{C}$

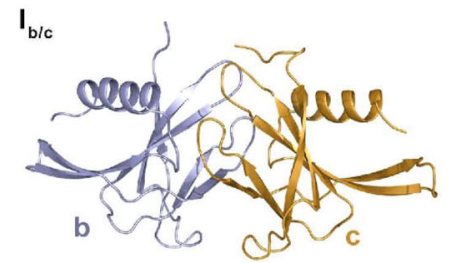

e

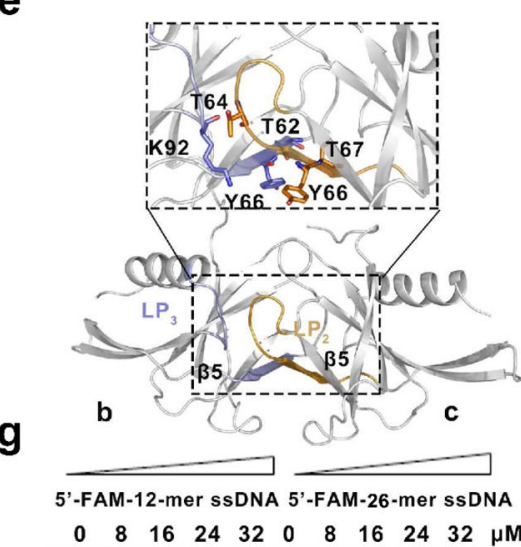

h

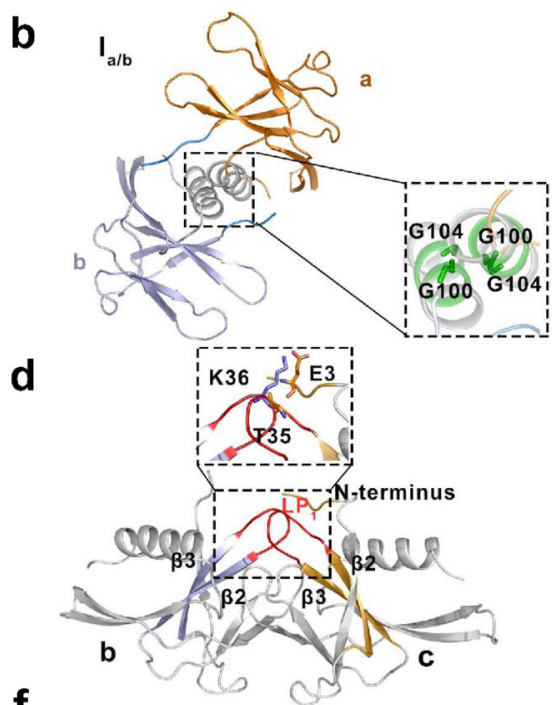

f

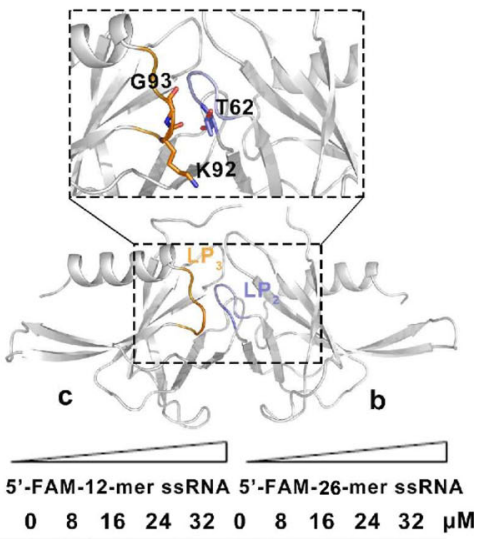

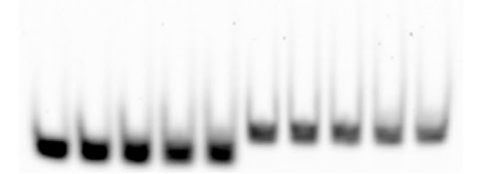

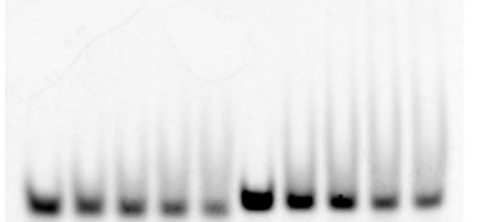

Fig. 3 Details of structure of the SARS-CoV-2 nsp9 tetramer and function in nucleic acid binding. $\mathbf{a}, \mathbf{b}$ Details of $I_{c / d}$ and $I_{a / b}$ in the helix interface. The molecules in these two interfaces are shown as cartoons and colored and labeled as in Fig. 2a. The expanded boxed area to the right shows a detailed view of the critical residues in the $I_{c / d}$ and $I_{a / b}$ interfaces, which are shown as sticks. c Molecules involved in $I_{b / c}$. The molecules in this interface are shown as cartoons and colored and labeled as in Fig. 2a. $\mathbf{d}-\mathbf{f}$ Detailed views of the first, second, and third contact regions in interface $\mathrm{I}_{\mathrm{b} / \mathrm{c}}$ respectively. The secondary structures of each contact region are colored and labeled according to Fig. 2a and the critical residues in each region are shown as sticks. g Single-stranded DNA (ssDNA)-binding abilities of SARS-CoV-2 nsp9, as determined by electrophoretic mobility shift assay. $\mathbf{h}$ Single-stranded RNA (ssRNA)-binding abilities of SARS-CoV-2 nsp9. The length of ssDNA, the state of SARS-CoV-2 nsp9, and the concentration of proteins used in the reaction system are indicated above the gel

Y66) on molecule c form four hydrogen bonds with residues $\mathrm{K} 92, \mathrm{~T} 67$, and Y66 on molecule b, respectively (Fig. 3e). The third contact region involved in interface $\mathrm{I}_{\mathrm{b} / \mathrm{c}}$ is composed of the $\mathrm{LP}_{3}$ loop on molecule $\mathrm{c}$ and the $\mathrm{LP}_{2}$ loop on molecule b. Residues K92 and G93 on molecule c form two hydrogen bonds with residue T62 on molecule b (Fig. 3f). These three contact surfaces in interface $\mathrm{I}_{\mathrm{b} / \mathrm{c}}$ contribute a hydrophobic base with eight hydrogen bonds and one salt bridge, making the SARS-
CoV-2 nsp9 tetramer extremely stable in the crystal structure.

\section{SARS-CoV-2 nsp9 with nucleic acid-binding ability}

Our previous research on nsp9 from IBV showed that the multimerization of this protein was necessary for protein-nucleic acid interaction [23]. In this present study, we observed the nucleic acid-binding ability of SARS-CoV-2 nsp9, using the electrophoretic mobility 
shift assay. Under favorable conditions, the nsp9 wild type was incubated with single-stranded nucleic acids. As shown in Fig. $3 g$ and h, the free single-stranded DNA and RNA bands in the lanes associated with nsp9 were slightly reduced with the increasing concentration of nsp9. Additionally, we observed the ability of nsp9 to interact with a range of sizes and types of singlestranded nucleic acids. Based on these results, we can conclude that nsp9 prefers to interact with the singlestranded nucleic acid.

\section{Discussion}

In this study, we first revealed the horseshoe-like tetrameric structure of nsp9 encoded by the genomic RNA of SARS-CoV-2. This homotetrameric structure comprised two significant styles of dimeric conformation that were found in nsp9 structures published in the last decade $[19,21]$. These structural results from independent research groups revealed that the dimerization of nsp9 might occur biologically and could play a pivotal role in the nucleic acid-binding function of the protein. However, because the dimerized nsp9 structure involves different dimeric forms, an interesting question is which of these is the major unit that interacts with nucleic acids. In the horseshoe-like tetrameric structure of SARS-CoV2 nsp9, we found that two dimeric forms exist. One of them, named the helix interface and first reported by Egloff et al. (2004) [21], is organized by the parallel association of the $\mathrm{C}$-terminal $\alpha$-helix and the $\mathrm{N}$-terminal loop. The second dimeric form, named the sheet interface and first mentioned by Sutton et al. (2004) [19], is formed by the zippering of each $\beta_{5}$ strand from both subunits. The sheet interface is much more stable in the tetrameric structure of SARS-CoV-2 nsp9 than in SARS$\mathrm{CoV}$ nsp9. Aside from the $\beta 5$ strand, there are three loops $\left(\mathrm{LP}_{1}, \mathrm{LP}_{2}\right.$, and $\left.\mathrm{LP}_{3}\right)$ involved in the nsp9 tetrameric interface. Therefore, we propose that these two dimeric forms are the fundamental units for building the highorder oligomers of nsp9. According to two different research groups that studied nsp9 mutants, the mutation of conserved residues located on the C-terminal $\alpha$-helix (e.g., SARS-CoV nsp9 G104E (PDB: 3EE7)) or the unexpected appearance of a disulfide on $\beta_{5}(\mathrm{hCoV}-229 \mathrm{E}$ nsp9 wild type (PDB: 2 J97)) could significantly change the dimer interface of nsp9 and its ability to bind with DNA or RNA $[20,22]$. In summary, the two dimeric interfaces that occurred in all crystal structures of nsp 9 are reasonable and likely contribute to the formation of the higher oligomeric states of the protein.

\section{Materials and methods}

Plasmid construction, protein expression, and purification The SARS-CoV-2 nsp 9 gene (genome nucleotides 12, 686-13,024; Gene ID: 43740578) was synthesized and cloned into the pET-28a (+)-SUMO vector using BamHI and XhoI sites (Qingke Biotech, China). The recombinant plasmids, which were confirmed by DNA sequencing (Sangon Biotech, China), were then used to transform Escherichia coli BL21 (DE3) (Transgene, China). Cells were cultured in Luria broth medium at $37^{\circ} \mathrm{C}$ until the optical density at $600 \mathrm{~nm}$ reached $0.6-0.8$. Protein expression was then induced by incubating the cells with $0.5 \mathrm{mM}$ isopropyl $\beta$-D-1-thiogalactopyranoside at $16^{\circ} \mathrm{C}$ for $18 \mathrm{~h}$. The nsp9 protein was labeled with a HisSUMO tag with a Ulp1 cleavage site, purified by nickelnitrilotriacetic acid affinity chromatography (Qiagen, Germany), and then cleaved with Ulp1 protease. The cleaved and tag-removed protein was loaded onto a Resource S chromatography column (GE Healthcare, USA) and eluted with a linear gradient of $10 \mathrm{mM}$ to $1 \mathrm{M} \mathrm{NaCl}$. The peak fraction containing the target protein was pooled, then concentrated to $1 \mathrm{~mL}$ using Amicon Ultra concentrators (cutoff size of $10 \mathrm{kDa}$; Millipore, USA), and finally loaded onto a Superdex 200 column (GE Healthcare, USA) for further purification with a buffer composed of $20 \mathrm{mM}$ Tris (pH 8.0), $150 \mathrm{mM} \mathrm{NaCl}, 1 \mathrm{mM}$ dithiothreitol, and $10 \%$ glycerol $(\mathrm{v} / \mathrm{v})$. The purity of the proteins was greater than $95 \%$, as confirmed by sodium dodecyl sulfate polyacrylamide gel electrophoresis (Supplementary Fig. 2a and 2b).

\section{Protein crystallization and optimization}

Using the hanging-drop vapor diffusion method, the nsp9 protein was quantified using absorbance readings at A280 nm (Thermo NanoDrop 2000, USA) and concentrated to $17 \mathrm{mg} / \mathrm{mL}$ for the crystallization trials, by mixing $1 \mu \mathrm{L}$ of protein with $1 \mu \mathrm{L}$ of reservoir solution at $20^{\circ} \mathrm{C}$. Commercial crystallization kits (Hampton Research, UK) were used to obtain the initial crystallization conditions. The protein crystals were obtained in a solution containing $100 \mathrm{mM}$ citric acid (pH 3.0), $200 \mathrm{mM}$ potassium sodium tartrate tetrahydrate, and $2.0 \mathrm{M}$ $\left(\mathrm{NH}_{4}\right)_{2} \mathrm{SO}_{4}$ after growth for 2 days at $20^{\circ} \mathrm{C}$ (Supplementary Fig. $2 \mathrm{c}$ and $2 \mathrm{~d}$ ). The crystals were then transferred to a solution containing $100 \mathrm{mM}$ citric acid ( $\mathrm{pH} 3.0$ ), $200 \mathrm{mM}$ sodium metformin, and $2.5 \mathrm{M}\left(\mathrm{NH}_{4}\right)_{2} \mathrm{SO}_{4}$, and the mixture was then frozen and stored in liquid nitrogen for future data collection.

\section{Data collection, processing, and structure determination} The nsp9 crystal diffraction data were collected at $196^{\circ} \mathrm{C}$, using the SSRF Beamline BL18U1 apparatus (Shanghai, China) at a wavelength of $0.97930 \AA$. Data were processed and scaled using the HKL2000 package (Supplementary Fig. 2e) [28]. The nsp9 structure was solved by Phaser in the CCP4 program suite using molecular replacement with the structure of SARS-CoV nsp9 RNA-replicase (PDB: 1QZ8) as an initial search 
model [29]. Cycles of refinement and model building were carried out using the REFMAC5, Phenix, and COOT software programs $[30,31]$. Model geometry was verified using MolProbity. The single crystals of SARS-CoV-2 nsp9 are in space group $C 222_{1}$, with cell dimensions of $a=88.7 \AA, b=134.5 \AA, c=167.0 \AA$, and $a=\beta=\gamma=90^{\circ}$. Both the Matthews coefficient estimation and the self-rotation function suggested the presence of six molecules per asymmetric unit. The final structure was refined to $2.95 \AA$ resolution. The final $R_{\text {work }}$ and $R_{\text {free }}$ for the refined structure were $21.3 \%$ and $29.9 \%$, respectively. In total, $96.1 \%$ of the amino acid residues fell in the most-favored region and the additionally allowed region of the Ramachandran plot. The structural figures were drawn using PyMOL [32]. The data collection and refinement statistics are shown in Table 1.

\section{Electrophoretic mobility shift assay}

The 5'-FAM-labeled 12-mer single-stranded DNA oligonucleotide 5'-GCTTTGATTTCG-3'， 5'-FAM-labeled 26-mer single-stranded DNA oligonucleotide 5'-GCTT TGATTTCGTGCATCTATGGAGC-3', 5'-FAM-labeled 12-mer single-stranded RNA oligonucleotide 5' GCUUUGAUUUCG-3' and 5'-FAM-labeled 26-mer single-stranded RNA oligonucleotide 5'-GCUUUG AUUUCGUGCAUCUAUGGAGC-3' were used for the electrophoretic mobility shift assay. Initially, $2 \mathrm{nmol}$ DNA was incubated with different concentrations of nsp9 protein (in the native), in a solution containing 10 mM 4-(2-hydroxyethyl)-1-piperazineethanesulfonic acid (pH 8.0), $50 \mathrm{mM} \mathrm{KCl}, 1 \mathrm{mM}$ ethylenediaminetetraacetic acid (EDTA), $0.05 \%$ Triton-X-100, and 5\% glycerol, for $30 \mathrm{~min}$ at $4{ }^{\circ} \mathrm{C}$. Thereafter, a $10 \times$ loading buffer $(250 \mathrm{mM}$ Tris- $\mathrm{HCl}(\mathrm{pH} 7.9)$ and $40 \%$ glycerol) was added to the

Table 1 SARS-CoV-2 nsp9 data collection and refinement statistics

\begin{tabular}{|c|c|}
\hline & SARS-CoV-2 nsp9 \\
\hline \multicolumn{2}{|l|}{ Data collection } \\
\hline Resolution $(\AA)$ & 2.95 \\
\hline Space group & $C 222_{1}$ \\
\hline Unit-cell parameters $\left(\AA^{\circ}{ }^{\circ}\right)$ & $a=88.7, b=134.5 c=167.0, a=\beta=\gamma=90$ \\
\hline Resolution $(\AA ̊)$ & $50.00-2.95(3.04-2.95)$ \\
\hline $\mathrm{R}_{\text {merge }}{ }^{\mathrm{a}}(\%)$ & $22.1(59.3)$ \\
\hline $\mathrm{R}_{\text {pim }} \mathrm{b}(\%)$ & $6.6(17.0)$ \\
\hline Average $\mid / \sigma(I)$ & $11.6(4.5)$ \\
\hline No. of observed reflections & $40,346(3400)$ \\
\hline No. of unique reflections & $21,270(1762)$ \\
\hline Completeness (\%) & $99.55(97.3)$ \\
\hline Multiplicity & $12.4(2.3)$ \\
\hline Matthews coefficient $\left(\AA^{3} \mathrm{Da}^{-1}\right)$ & 3.26 \\
\hline Solvent content (\%) & 62.3 \\
\hline Molecules per asymmetric unit & 6 \\
\hline \multicolumn{2}{|l|}{ Refinement } \\
\hline Resolution $(\AA)$ & $44.49-2.95$ \\
\hline$R_{\text {work }} / R_{\text {free }}$ & $0.21 / 0.30$ \\
\hline Ramachandran favored (\%) & 88.31 \\
\hline Ramachandran outliers (\%) & 3.32 \\
\hline \multicolumn{2}{|l|}{ No. of atoms } \\
\hline Protein & 4963 \\
\hline Water & 66 \\
\hline Wilson B value & 87.32 \\
\hline \multicolumn{2}{|l|}{ Root-mean-square deviations } \\
\hline Bond length $(\AA)$ & 0.010 \\
\hline Bond angle $\left(^{\circ}\right)$ & 1.293 \\
\hline
\end{tabular}

${ }^{\mathrm{a}} R_{\text {merge }}=\Sigma_{h k l} \Sigma_{i}\left|l_{i}(h k l)-\langle I(h k l)\rangle\right| / \Sigma_{h k \mid} \Sigma_{i} l_{i}(h k l)$, where $l_{i}(h k l)$ is an individual intensity measurement and $\langle I(h k l)\rangle$ is the average intensity for all $i$ reflections

${ }^{\mathrm{b}} R_{\text {pim }}$ is approximately estimated by multiplying the $\mathrm{R}_{\text {merge }}$ value by the factor $[1 /(N-1)]^{1 / 2}$, where $N$ is the overall redundancy of the data set 
mixture. Then, samples were electrophoresed on $6.5 \%$ non-denaturing Tris-borate-EDTA (TBE) polyacrylamide gels for $40 \mathrm{~min}$ at a voltage of $100 \mathrm{~V}$, and the results were determined with a ChemiDoc Touch Imaging system (Bio-Rad, USA).

\section{Protein data Bank accession codes}

The structure factors and atomic coordinates have been deposited in the Protein Data Bank under the PDB ID codes $7 \mathrm{BWQ}$.

\section{Supplementary information}

Supplementary information accompanies this paper at https://doi.org/10 1186/s43556-020-00005-0

Additional file 1: Figure S1. The OB-fold cluster of SARS-CoV-2 nsp9. (a) The different layers are colored orange, light blue, and pale green, respectively, and protomers in every layer are depicted in cartoon representations and labeled with uppercase letters. (b) Molecules $d^{\prime}, c^{\prime}$, $a_{\text {sym }}$, and $b_{\text {sym }}$ in the stable tetramer are colored in bright orange, pale green, light blue, and orange, respectively, and depicted in cartoon and surface representations.

Additional file 2: Figure S2. Purification and X-ray data collection of SARS-CoV-2 nsp9. (a) The chromatography of the purification of SARSCoV-1 nsp9 with a Superdex200 10 TM 1000 column. (b) SDS-PAGE analysis result of SARS-CoV-2 nsp9. M is the protein marker. (c) The primary crystal state of SARS-CoV-2 nsp9 in the commercial crystallization kit. (d) The crystal for X-ray data collection of SARS-CoV-2 nsp9 after crystallization condition optimized. (e) X-ray diffraction pattern for structure analysis (left) and X-ray diffraction point atlas of SARS-CoV-2 nsp9 (right).

Additional file 3: Table S1. PDBePISA calculation of interface parameters of the quaternary structure of SARS-CoV-2 nsp9.

\section{Abbreviations}

CoV: Coronavirus; COVID-19: Coronavirus disease 2019; hCoV: Human coronavirus; LP: Loop protein; OB-Fold superfamily: oligosaccharide/ oligonucleotide-binding superfamily; nsp9: Nonstructural protein 9; SARSCoV-2: Severe acute respiratory syndrome coronavirus-2

\section{Acknowledgments}

We sincerely appreciate the staff of the State Key Laboratory of Biotherapy, Sichuan University, who assisted in our research work during the COVID-19 epidemic. The X-ray diffraction experiments were carried out at the Shanghai Synchrotron Radiation Facility (SSRF) BL18U1. We gratefully thank the beamline staff for their technical help and Dr. Qingxiang Sun for sharing with us the beamline schedule. We appreciate Dr. Guangwen Lu and Dr. Shengce Tao for providing us with the associated plasmids and Dr. Qiang Chen for gifting us valuable DNA. This work was supported by grants from the National Key Research and Development Program of China (2017YFA0505903, 2018YFC1312300), the research fund on COVID-19 of Sichuan Province (2020YFS0010), and the project on COVID-19 of West China Hospital, Sichuan University (HX-2019-nCoV-044).

\section{Code availability}

Not applicable.

\section{Authors' contributions}

DS and CZ designed the study. CZ purified and characterized the proteins, cultivated the protein crystals, collected X-ray diffraction data, and managed the biological experiments. DS solved the crystal structure. YY. assisted with the biological experiments and provided essential data for this study. DS and $Y C$ took the lead in writing the manuscript. LL designed and created all the Figs. YC, CC, and JH contributed to the data analysis and the final version of the manuscript. DS had final approval of the version to be published.

\section{Funding}

This work was supported by grants from the National Key Research and Development Program of China (2017YFA0505903, 2018YFC1312300), the research fund on COVID-19 of Sichuan Province (2020YFS0010), and the project on COVID-19 of West China Hospital, Sichuan University (HX-2019nCoV-044).

\section{Availability of data and materials}

All data generated or analysed during this study are included in this published article [and its supplementary information files].

\section{Ethics approval and consent to participate}

Not applicable.

\section{Consent for publication}

Not applicable.

\section{Competing interests}

Changhui Zhang, Yiping Chen, Li Li, Yan Yang, Jun He, Cheng Chen, and Dan Su declare that they have no conflicts of interest.

\section{Author details}

${ }^{1}$ State Key Laboratory of Biotherapy, West China Hospital, Sichuan University, and Collaborative Innovation Center for Biotherapy, Chengdu 610041, PR China. ${ }^{2}$ CAS Key Laboratory of Regenerative Biology, Guangzhou Institutes of Biomedicine and Health, Chinese Academy of Sciences, Guangzhou 510530, PR China. ${ }^{3}$ School of Life Sciences, Tianjin University, Tianjin 300072, PR China.

Received: 11 June 2020 Accepted: 3 August 2020

Published online: 20 August 2020

\section{References}

1. Coronaviridae Study Group of the International Committee on Taxonomy of V. The species severe acute respiratory syndrome-related coronavirus: classifying 2019-nCoV and naming it SARS-CoV-2. Nat Microbiol. 2020:5(4): 536-44. https://doi.org/10.1038/s41564-020-0695-z.

2. Zhu N, Zhang D, Wang W, Li X, Yang B, Song J, et al. A novel coronavirus from patients with pneumonia in China, 2019. N Engl J Med. 2020;382(8): 727-33. https://doi.org/10.1056/NEJMoa2001017.

3. Hui DS. E IA, Madani TA, Ntoumi F, Kock R, Dar O, et al. the continuing 2019-nCoV epidemic threat of novel coronaviruses to global health - the latest 2019 novel coronavirus outbreak in Wuhan, China. Int J Infect Dis. 2020;91:264-6. https://doi.org/10.1016/j.jij. 2020.01.009

4. WHO. WHO, Coronavirus disease (COVID-2019) situation reports. World Health Organization: Geneva, Switzerland. 2020. https://www.who.int/ emergencies/diseases/novel-coronavirus-2019/situation-reports of subordinate document. Accessed 27 July 2020

5. Ji W, Wang W, Zhao X, Zai J, Li X. Cross-species transmission of the newly identified coronavirus 2019-nCoV. J Med Virol. 2020;92(4):433-40. https://doi. org/10.1002/jmv.25682.

6. Lu R, Zhao X, Li J, Niu P, Yang B, Wu H, et al. Genomic characterisation and epidemiology of 2019 novel coronavirus: implications for virus origins and receptor binding. Lancet. 2020:395(10224):565-74. https://doi.org/10.1016/ S0140-6736(20)30251-8.

7. Zhang YZ, Holmes EC. A genomic perspective on the origin and emergence of SARS-CoV-2. Cell. 2020;181(2):223-7. https://doi.org/10.1016/j.cell.2020.03.035.

8. Han Q, Lin Q, Jin S, You L. Coronavirus 2019-nCoV: a brief perspective from the front line. J Inf Secur. 2020;80(4):373-7. https://doi.org/10.1016/j.jinf.2020. 02.010 .

9. Fehr AR, Perlman S. Coronaviruses: an overview of their replication and pathogenesis. Methods Mol Biol. 2015;1282:1-23. https://doi.org/10.1007/ 978-1-4939-2438-7_1.

10. Wang Q, Zhang Y, Wu L, Niu S, Song C, Zhang Z, et al. Structural and functional basis of SARS-CoV-2 entry by using human ACE2. Cell. 2020. https://doi.org/10.1016/j.cell.2020.03.045

11. van Hemert MJ, van den Worm SH, Knoops K, Mommaas AM, Gorbalenya AE, Snijder EJ. SARS-coronavirus replication/transcription complexes are membrane-protected and need a host factor for activity in vitro. PLOS Pathog. 2008;4(5):e1000054. https://doi.org/10.1371/journal.ppat.1000054. 
12. te Velthuis AJ, Arnold JJ, Cameron CE, van den Worm SH, Snijder EJ. The RNA polymerase activity of SARS-coronavirus nsp12 is primer dependent. Nucleic Acids Res. 2010;38(1):203-14. https://doi.org/10.1093/nar/gkp904.

13. Lee NR, Kwon HM, Park K, Oh S, Jeong YJ, Kim DE. Cooperative translocation enhances the unwinding of duplex DNA by SARS coronavirus helicase nsP13. Nucleic Acids Res. 2010;38(21):7626-36. https://doi.org/10. 1093/nar/gkq647.

14. Li S, Zhao Q, Zhang Y, Zhang Y, Bartlam M, Li X, et al. New nsp8 isoform suggests mechanism for tuning viral RNA synthesis. Protein Cell. 2010;1(2): 198-204. https://doi.org/10.1007/s13238-010-0028-8.

15. Bouvet M, Imbert I, Subissi L, Gluais L, Canard B, Decroly E. RNA 3'-end mismatch excision by the severe acute respiratory syndrome coronavirus nonstructural protein nsp10/nsp14 exoribonuclease complex. Proc Natl Acad Sci U S A. 2012;109(24):9372-7. https://doi.org/10.1073/pnas. 1201130109.

16. Joseph JS, Saikatendu KS, Subramanian V, Neuman BW, Buchmeier MJ, Stevens RC, et al. Crystal structure of a monomeric form of severe acute respiratory syndrome coronavirus endonuclease nsp15 suggests a role for hexamerization as an allosteric switch. J Virol. 2007;81(12):6700-8. https:// doi.org/10.1128/JVI.02817-06.

17. te Velthuis AJ, van den Worm SH, Snijder EJ. The SARS-coronavirus nsp7+ nsp8 complex is a unique multimeric RNA polymerase capable of both de novo initiation and primer extension. Nucleic Acids Res. 2012;40(4):1737-47. https://doi.org/10.1093/nar/gkr893.

18. Jin Z, Du X, Xu Y, Deng Y, Liu M, Zhao Y, et al. Structure of M (pro) from COVID-19 virus and discovery of its inhibitors. Nature. 2020. https://doi.org/ 10.1038/s41586-020-2223-y.

19. Sutton G, Fry E, Carter L, Sainsbury S, Walter T, Nettleship J, et al. The nsp9 replicase protein of SARS-coronavirus, structure and functional insights. Structure. 2004;12(2):341-53. https://doi.org/10.1016/j.str.2004.01.016.

20. Miknis ZJ, Donaldson EF, Umland TC, Rimmer RA, Baric RS, Schultz LW. Severe acute respiratory syndrome coronavirus nsp9 dimerization is essential for efficient viral growth. J Virol. 2009;83(7):3007-18. https://doi. org/10.1128/JVI.01505-08

21. Egloff MP, Ferron F, Campanacci V, Longhi S, Rancurel C, Dutartre H, et al. The severe acute respiratory syndrome-coronavirus replicative protein nsp9 is a single-stranded RNA-binding subunit unique in the RNA virus world. Proc Natl Acad Sci U S A. 2004;101(11):3792-6. https://doi.org/10.1073/pnas. 0307877101.

22. Ponnusamy R, Moll R, Weimar T, Mesters JR, Hilgenfeld R. Variable oligomerization modes in coronavirus non-structural protein 9. J Mol Biol. 2008:383(5):1081-96. https://doi.org/10.1016/j.jmb.2008.07.071.

23. Hu T, Chen C, Li H, Dou Y, Zhou M, Lu D, et al. Structural basis for dimerization and RNA binding of avian infectious bronchitis virus nsp9. Protein Sci. 2017;26(5):1037-48. https://doi.org/10.1002/pro.3150.

24. Zeng Z, Deng F, Shi K, Ye G, Wang G, Fang L, et al. Dimerization of coronavirus nsp9 with diverse modes enhances its nucleic acid binding affinity. J Virol. 2018;92(17). https://doi.org/10.1128/JVl.00692-18

25. Murzin AG. OB (oligonucleotide/oligosaccharide binding)-fold: common structural and functional solution for non-homologous sequences. EMBO J. 1993;12(3):861-7.

26. Theobald DL, Mitton-Fry RM, Wuttke DS. Nucleic acid recognition by OBfold proteins. Annu Rev Biophys Biomol Struct. 2003;32:115-33. https://doi. org/10.1146/annurev.biophys.32.110601.142506.

27. Krissinel $E$, Henrick K. Inference of macromolecular assemblies from crystalline state. J Mol Biol. 2007;372(3):774-97. https://doi.org/10.1016/j.jmb.2007.05.022.

28. Otwinowski Z, Minor W. Processing of X-ray diffraction data collected in oscillation mode. Methods Enzymol. 1997;276:307-26.

29. Winn MD, Ballard CC, Cowtan KD, Dodson EJ, Emsley P, Evans PR, et al. Overview of the CCP4 suite and current developments. Acta Crystallogr D Biol Crystallogr. 2011;67(Pt 4):235-42. https://doi.org/10.1107/S0907444910045749.

30. Adams PD, Afonine PV, Bunkoczi G, Chen VB, Davis IW, Echols N, et al. PHEN IX: a comprehensive python-based system for macromolecular structure solution. Acta Crystallogr D Biol Crystallogr. 2010;66(Pt 2):213-21. https://doi. org/10.1107/S0907444909052925.

31. McCoy AJ, Grosse-Kunstleve RW, Adams PD, Winn MD, Storoni LC, Read RJ. Phaser crystallographic software. J Appl Crystallogr. 2007:40(Pt 4):658-74 https://doi.org/10.1107/S0021889807021206.

32. Rigsby RE, Parker AB. Using the PyMOL application to reinforce visual understanding of protein structure. Biochem Mol Biol Educ. 2016;44(5):4337. https://doi.org/10.1002/bmb.20966.

\section{Publisher's Note}

Springer Nature remains neutral with regard to jurisdictional claims in published maps and institutional affiliations. 